\title{
ON MINIMAL HYPERSURFACES WITH CONSTANT SCALAR CURVATURES IN $S^{4}$
}

\author{
SHAOPING CHANG
}

\section{Introduction}

Let $M^{n}$ be a piece of minimally immersed hypersurface in the unit sphere $S^{n+1}$, and $h$ its second fundamental form. Denote by $R$ and $S$ its scalar curvature and the square norm of $h$, respectively. It is well known that $S=n(n-1)-R$ from the structure equations of both $M^{n}$ and $S^{n+1}$. In 1968, J. Simons [9] observed that if $S \leq n$ everywhere and either $M^{n}$ is compact or $S$ is constant, then $S \in\{0, n\}$. Clearly, $M^{n}$ is contained in an equatorial sphere if $S=0$. And when $S=n, M^{n}$ is indeed a piece of a product of spheres, due to the works of Chern, do Carmo, and Kobayashi [4] and Lawson [6]. These two kinds of hypersurfaces are the so-called isoparametric ones of types 1 and 2, respectively.

Definition. A hypersurface of $S^{n+1}$ is called isoparametric of type $g$ if it has $g$ distinct constant principal curvatures of constant multiplicities.

The classification of isoparametric hypersurfaces in spheres is far from being completed although the study has been very fruitful. An interested reader is referred to the book of Cecil and Ryan [2]. Here we will only mention a pioneering work of E. Cartan [1] and leave our pursuit on this topic in [3].

Theorem [Cartan, 1939]. There exist minimal isoparametric hypersurfaces of type 3 in spheres only in the dimension of 3,6,12, and 24. Moreover, it is unique in each of such dimensions up to a rotation on the sphere.

These hypersurfaces will be referred to as Cartan's minimal hypersurfaces.

We are concerned about the following conjecture posed by Chern [11].

Chern Conjecture. For any $n \geq 3$, the set $R_{n}$ of all the real numbers each of which can be realized as the constant scalar curvature of a closed minimally immersed hypersurface in $S^{n+1}$ is discrete.

There have been many works in this regard (e.g. [5], [7], [8], [10]). In the special case of $n=3$, Peng and Terng [7], [8] derived the following

Received February 26, 1992. 
Theorem [Peng-Terng, 1983]. If $M^{3}$ is a closed minimally immersed hypersurface with constant scalar curvature $R \leq 3$ in $S^{4}$, then either $R=3$ or $R \leq 0$.

Moreover, if $M^{3}$ in addition has only simple principal curvatures everywhere, then $M^{3}$ is a Cartan's minimal hypersurface. In particular, $R=0$.

Remark. The second statement stated here appears to be slightly stronger than their original one but follows directly from their proof.

Recently, De Almeida and Brito [5] exhibited the following theorem

Theorem [De Almeida-Brito, 1990]. If $M^{3}$ is a closed minimally immersed hypersurface with constant scalar curvature $R \geq 0$ in $S^{4}$, then $M^{3}$ is isoparametric.

In the present paper, we will give an affirmative answer to Chern conjecture when $n=3$ by settling the complementary case of the Peng-Terng theorem. Namely, we will establish

Main Theorem. If $M^{3}$ is a piece of minimally immersed hypersurface with constant scalar curvature $R$ in $S^{4}$ and has multiple principal curvatures somewhere, then either $R=3$, or 6 .

Consequently, combining the Main Theorem with the works of Cartan, Simons, Chern, do Carmo, and Kobayashi, and Peng and Terng, we have the following

Classification Theorem. A closed minimally immersed hypersurface with constant scalar curvature in $S^{4}$ is either an equatorial 3-sphere, a product of spheres, or a Cartan's minimal hypersurface.

In particular, $R_{n}=\{0,3,6\}$.

Remark. Note that both our Main Theorem and the uniqueness theorem of Chern, do Carmo, and Kobayashi cited at the beginning are valid without assuming the compactness of $M^{3}$. Our result also establishes the following conjecture of Robert Bryant in the special case where $M^{3}$ has multiple principal curvatures somewhere.

Bryant Conjecture. A piece of minimally immersed hypersurface of constant scalar curvature in $S^{4}$ is isoparametric.

This conjecture is still open in general for there has been no local version of the Peng-Terng theorem.

We will first present some terminology and set up notation in $\S 1$, and the proof of the Main Theorem will be given in $\S 2$.

Unless otherwise indicated, the summation convention applies throughout this paper. And we will always use $i, j, k, \ldots$, for indices running over $\{1,2,3\}$ and $A, B, C, \ldots$, over $\{1,2,3,4\} ; \delta_{A B}$ denotes the Kronecker symbol. 


\section{Terminology and notation}

Let $M^{3}$ be a manifold of dimension 3 immersed in a Riemannian manifold $N^{4}$ of dimension 4. Choose a local orthonormal frame field $\left\{e_{A}\right\}$ in $N^{4}$ such that, after restriction to $M^{3}$, the $e_{j}$ 's are tangent to $M^{3}$. Denote the dual coframe by $\left\{\omega_{A}\right\}$. Then the structure equations of $N^{4}$ are given by

$$
\begin{aligned}
d \omega_{A} & =\omega_{A B} \wedge \omega_{B}, \quad \omega_{A B}+\omega_{B A}=0, \\
d \omega_{A B} & =\omega_{A C} \wedge \omega_{C B}-\frac{1}{2} K_{A B C D} \omega_{C} \wedge \omega_{D}, \quad K_{A B C D}+K_{A B D C}=0 .
\end{aligned}
$$

We call $K_{A B C D}$, its contractions $K_{A C}=K_{A B C B}$ and $K=K_{A B A B}$, respectively, the curvature tensor, the Ricci curvature tensor and the scalar curvature of $N^{4}$.

When $N^{4}$ is the unit sphere $S^{4}$, it turns out that

$$
K_{A B C D}=\delta_{A C} \delta_{B D}-\delta_{A D} \delta_{B C}
$$

Next, we restrict all tensors to $M^{3}$. First of all, $\omega_{4}=0$ on $M^{3}$. Then $\omega_{4 i} \wedge \omega_{i}=d \omega_{4}=0$. By Cartan's lemma, we can write

$$
\omega_{4 i}=h_{i j} \omega_{j}, \quad \text { with } h_{i j}=h_{j i} \text {. }
$$

We call $h=\sum_{i, j} h_{i j} \omega_{i} \omega_{j}$, the eigenvalues $\lambda_{i}$ of matrix $\left(h_{i j}\right)$, and $H=$ $\sum_{i} h_{i i}=\sum_{i} \lambda_{i}$, respectively, the second fundamental form, the principal curvatures, and the mean curvature of $M^{3}$. And $M^{3}$ is said to be minimal if $H$ vanishes identically.

Second, from

$$
\begin{aligned}
d \omega_{i} & =\omega_{i j} \wedge \omega_{j}, \quad \omega_{j j}+\omega_{j i}=0, \\
d \omega_{i j} & =\omega_{i k} \wedge \omega_{k j}-\frac{1}{2} R_{i j k l} \omega_{k} \wedge \omega_{l}
\end{aligned}
$$

we find the curvature tensor of $M^{3}$ is

$$
R_{i j k l}=K_{i j k l}+h_{i k} h_{j l}-h_{i l} h_{j k} .
$$

Therefore, if $M^{3}$ is minimal, its Ricci curvature tensor and scalar curvature are given by, respectively,

$$
R_{i k}=2 \delta_{i k}-h_{i j} h_{j k}, \quad R=6-S,
$$

where $S=\sum_{i, j} h_{i j}^{2}$ is the square norm of $h$. 
Given a symmetric 2-tensor $T=T_{i j} \omega_{i} \omega_{j}$ on $M^{3}$, we also define its covariant derivatives, denoted by $\nabla T, \nabla^{2} T$ and $\nabla^{3} T$, etc. with components $T_{i j, k}, T_{i j, k l}$ and $T_{i j, k l p}$, respectively, as follows:

$$
\begin{aligned}
T_{i j, k} \omega_{k} & =d T_{i j}+T_{s j} \omega_{s i}+T_{i s} \omega_{s j}, \\
T_{i j, k l} \omega_{l} & =d T_{i j, k}+T_{s j, k} \omega_{s i}+T_{i s, k} \omega_{s j}+T_{i j, s} \omega_{s k}, \\
T_{i j, k l p} \omega_{p} & =d T_{i j, k l}+T_{s j, k l} \omega_{s i}+T_{i s, k l} \omega_{s j}+T_{i j, s l} \omega_{s k}+T_{i j, k s} \omega_{s l}, \quad \text { etc. }
\end{aligned}
$$

In the next section, we sometimes also use $\nabla_{e_{k}} T_{i j}$ to denote $T_{i j, k}$, etc.

Example 1. $T=\sum_{i} \omega_{i}^{2}$, i.e., $T_{i j}=\delta_{i j}$.

Since $d \delta_{i j}=0$ and $\delta_{s j} \omega_{s i}+\delta_{i s} \omega_{s j}=\omega_{j i}+\omega_{i j}=0$, we find

$$
\nabla T=0, \quad \text { i.e., } \delta_{i j, k}=0, \quad \forall i, j, k \text {. }
$$

In general, the resulting tensors are no longer symmetric, and the rules to switch sub-indices obey the Ricci formulas as follows:

$$
\begin{aligned}
T_{i j, k l}-T_{i j, l k} & =T_{s j} R_{s i k l}+T_{i s} R_{s j k l}, \\
T_{i j, k l p}-T_{i j, k p l}= & T_{s j, k} R_{s i l p}+T_{i s, k} R_{s j l p}+T_{i j, s} R_{s k l p}, \\
T_{i j, k l p m}-T_{i j, k l m p}= & T_{s j, k l} R_{s i p m} \\
& +T_{i s, k l} R_{s j p m}+T_{i j, s l} R_{s k p m}+T_{i j, k s} R_{s l p m}, \quad \text { etc. }
\end{aligned}
$$

Example 2. $T=h-h_{i j} \omega_{i} \omega_{j}$ with $N^{4}=S^{4}$.

For the sake of simplicity, we always omit the comma $($,$) between$ indices in this special case.

Recall that $\omega_{4 i}=h_{i j} \omega_{j}$, and $d \omega_{4 i}=\omega_{4 C} \wedge \omega_{C i}-\frac{1}{2} K_{4 i C D} \omega_{C} \wedge \omega_{D}$. Since $K_{4 i C D} \omega_{C} \wedge \omega_{D}=0$ on $M^{3}$ when $N^{4}=S^{4}$, we find

$$
d\left(h_{i j} \omega_{j}\right)=h_{j k} \omega_{k} \wedge \omega_{j i} .
$$

Therefore,

$$
h_{i j k} \omega_{k} \wedge \omega_{j}=\left(d h_{i j}+h_{k j} \omega_{k i}+h_{i k} \omega_{k j}\right) \wedge \omega_{j}=0 ;
$$

i.e., $h_{i j k}$ is symmetric in all the indices. Moreover, in the case that $M^{3}$ is minimal, we have

$$
\begin{aligned}
h_{i j k k} & =h_{k i j k}=h_{k i k j}+h_{m i} R_{m k j k}+h_{k m} R_{m i j k} \\
& =h_{m i}\left(2 \delta_{m j}-h_{m k} h_{k j}\right)+h_{k m}\left(\delta_{m j} \delta_{i k}-\delta_{m k} \delta_{i j}+h_{m j} h_{i k}-h_{m k} h_{i j}\right) \\
& =3 h_{i j}-h_{k m} h_{m k} h_{i j} .
\end{aligned}
$$

It follows that

$$
\frac{1}{2} \Delta S=(3-S) S+\sum_{i, j, k} h_{i j k}^{2}
$$


We finish the current section by noting the following combination formula

$$
\sum_{i, j, k}=6 \sum_{i, j, k \text { distinct }}+\sum_{i=j=k}+3 \sum_{i=j \neq k}
$$

provided the summand is symmetric in the indices $i, j$, and $k$. It will be applied in the next section whenever we want to compute a summation explicitly.

\section{Proof of the Main Theorem}

By virtue of the result of Simons, we only need to show that $S=3$ if $S \geq 3$ and $M^{3}$ has multiple principal curvatures at some point, say, $p \in \bar{M}^{3}$. The idea is to investigate the second fundamental form $h$ and its covariant derivatives at $p$.

Let $\lambda_{i}, i=1,2,3$, be the principal curvature functions of $M^{3}$.

Now suppose that at $p \in M^{3}$,

$$
\lambda_{1}=\lambda_{2} \quad(=\lambda)
$$

By the minimality of $M^{3}$,

$$
\lambda_{3}=-2 \lambda \text {. }
$$

Thus, we may assume that at $p$,

$$
\left(h_{i j}\right)=\left(\begin{array}{lll}
\lambda & & \\
& \lambda & \\
& & -2 \lambda
\end{array}\right),
$$

where $\lambda^{2}=\frac{S}{6} \geq \frac{1}{2}$ since $\sum_{i} \lambda_{i}^{2}=S$.

We will next study the covariant derivative $\nabla h$ of $h$ at $p$. From $\sum_{i, j} h_{i j}^{2}=$ const.

$$
h_{i j} h_{i j k}=0, \quad \forall k
$$

It follows that, at $p$,

$$
\lambda_{1} h_{11 k}+\lambda_{2} h_{22 k}+\lambda_{3} h_{33 k}=0 \text {. }
$$

Note that $h_{11 k}+h_{22 k}+h_{33 k}=0, \forall k$, everywhere; we solve that, at $p$,

$$
h_{22 k}=-h_{11 k}, \quad h_{33 k}=0, \quad \forall k \text {. }
$$

Furthermore, since at $p,\left\{e_{1}, e_{2}\right\}$ is a basis of the $\lambda$-eigenspace of $\left(h_{i j}\right)$, we may rotate it if necessary to have

$$
h_{123}(p)=0 \text {. }
$$


Recall that $h_{i j k}$ is symmetric over $\{i, j, k\}$, and by $(\mathbf{S})$ in $\S 1$

$$
\sum_{i, j, k} h_{i j k}^{2}=S(S-3) \text { everywhere. }
$$

We compute that at $p$

$$
\begin{aligned}
\sum_{i, j, k} h_{i j k}^{2} & =6 h_{123}^{2}+\sum_{i} h_{i i i}^{2}+3 \sum_{i \neq k} h_{i i k}^{2} \\
& =\left(h_{111}^{2}+h_{222}^{2}\right)+3\left(h_{112}^{2}+h_{113}^{2}+h_{221}^{2}+h_{223}^{2}\right) \\
& =6 a+4 b,
\end{aligned}
$$

where $a=h_{113}^{2}(p) \geq 0, b=h_{111}^{2}(p)+h_{112}^{2}(p) \geq 0$.

Hence,

$$
6 a+4 b=S(S-3) .
$$

It therefore suffices to show that $a=b=0$. We will achieve this by studying the higher covariant derivatives of $h$.

Let us start with the following

Lemma 1. At the point $p$,

(1) $h_{i j k l}$ is symmetric in all the indices except when $\{i, j, k, l\}$ is a permutation of either $\{1,1,3,3\}$ or $\{2,2,3,3\}$.

(2) $h_{3311}=h_{3322}=\frac{2}{3 \lambda}(a+b), h_{3333}=\frac{2 a}{3 \lambda}, h_{3312}=0, h_{3313}=\frac{2}{3 \lambda} h_{111} h_{113}$, $h_{3323}=\frac{2}{3 \lambda} h_{112} h_{113}$, and $h_{1111}=h_{2222}, h_{1133}=h_{2233}=-\frac{a}{3 \lambda}$.

Proof. The first assertion simply follows from the Ricci formula

$$
\begin{aligned}
h_{i j k l}-h_{i j l k} & =h_{s j} R_{s i k l}+h_{i s} R_{s j k l} \\
& =\left(\lambda_{i}-\lambda_{j}\right)\left(1+\lambda_{i} \lambda_{j}\right)\left(\delta_{i k} \delta_{j l}-\delta_{i l} \delta_{j k}\right), \quad \forall i, j, k, l .
\end{aligned}
$$

To see (2), we employ $h_{i j k} h_{i j l}+h_{i j} h_{i j k l}=0, \forall k, l$, everywhere from $\sum_{i, j} h_{i j}^{2}=$ const. By evaluating the above equation at $p$, we have

$$
h_{i j k} h_{i j l}+\lambda\left[\left(h_{11 k l}+h_{22 k l}\right)-2 h_{33 k l}\right]=0 \text {. }
$$

Now, since $h_{11 k l}+h_{22 k l}+h_{33 k l}=0, \forall k, l$, everywhere, it reads

$$
3 \lambda h_{33 k l}=h_{i j k} h_{i j l}, \quad \text { at } p, \forall k, l \text {. }
$$

This yields the first 6 equalities by explicitly writing out all terms on the right-hand side for all pairs $(k, l)$. And in turn, we find $h_{1111}=$ $-h_{2211}-h_{3311}=-h_{1122}-h_{3322}=h_{2222}$.

Finally, by the Ricci formula we compute that

$$
\begin{aligned}
h_{1133} & =h_{1313}=h_{1331}+\left(\lambda_{1}-\lambda_{3}\right)\left(1+\lambda_{1} \lambda_{3}\right) \\
& =\frac{2}{3 \lambda}(a+b)+3 \lambda\left(1-2 \lambda^{2}\right) \\
& =-\frac{a}{3 \lambda},
\end{aligned}
$$


where in the last equality we use $3 \lambda\left(2 \lambda^{2}-1\right)=\frac{1}{6 \lambda} S(S-3)=\frac{1}{3 \lambda}(3 a+2 b)$.

Similarly, $h_{2233}=-\frac{a}{3 \lambda}$ follows in the same way, or from $h_{2233}=$ $-\left(h_{1133}+h_{3333}\right)$.

This proves Lemma 1.

Lemma 2. At $p$,

$$
x+2 y=\frac{26}{9} a^{2}+\frac{7}{18} a b-b^{2}-\frac{5}{4} S b,
$$

where $x=\lambda^{2}\left[3\left(h_{1123}^{2}+h_{2213}^{2}\right)+h_{1113}^{2}+h_{2223}^{2}\right]$ and $y=\lambda^{2}\left(h_{1111}^{2}+h_{1112}^{2}\right)+$ $(a+b) \lambda h_{1111}$.

Proof. It is shown in [7] that

$$
\sum_{i, j, k, l} h_{i j k l}^{2}=3 S(S-3)^{2}-\frac{5}{3} \sum_{i, j, k} h_{i j k}^{2}\left(\lambda_{i}+\lambda_{j}+\lambda_{k}\right)^{2} .
$$

Now at $p$,

$$
\begin{aligned}
\sum_{i, j, k} h_{i j k}^{2}\left(\lambda_{i}+\lambda_{j}+\lambda_{k}\right)^{2} & =3 \sum_{i \neq k} h_{i i k}^{2}\left(2 \lambda_{i}+\lambda_{k}\right)^{2}+\sum_{i} h_{i i i}^{2}\left(3 \lambda_{i}\right)^{2} \\
& =3\left(h_{112}^{2}+h_{221}^{2}\right)(3 \lambda)^{2}+\left(h_{111}^{2}+h_{222}^{2}\right)(3 \lambda)^{2} \\
& =36 \lambda^{2} b .
\end{aligned}
$$

Hence,

$$
\text { RHS of }(*)=3 S(S-3)^{2}-10 S b .
$$

Moreover, since

$$
\begin{aligned}
\sum_{i, j, k} h_{i j k 1}^{2}= & 6 h_{1231}^{2}+\sum_{i} h_{i i i 1}^{2}+3 \sum_{i \neq k} h_{i i k 1}^{2} \\
= & 6 h_{1231}^{2}+h_{1111}^{2}+h_{2221}^{2}+h_{3331}^{2} \\
& +3\left(h_{1121}^{2}+h_{1131}^{2}+h_{2211}^{2}+h_{2231}^{2}+h_{3311}^{2}\right) \\
= & 3\left(2 h_{1123}^{2}+h_{1113}^{2}+h_{2213}^{2}\right)+4\left(h_{1111}^{2}+h_{1112}^{2}\right) \\
& +h_{3331}^{2}+6\left(h_{1111} h_{3311}+h_{3311}^{2}\right), \\
\sum_{i, j, k} h_{i j k 2}^{2}= & 6 h_{1232}^{2}+\sum_{i} h_{i i i 2}^{2}+3 \sum_{i \neq k} h_{i i k 2}^{2} \\
= & 6 h_{2213}^{2}+h_{1112}^{2}+h_{2222}^{2}+h_{3332}^{2} \\
& +3\left(h_{1122}^{2}+h_{1132}^{2}+h_{2212}^{2}+h_{2232}^{2}+h_{3322}^{2}\right) \\
= & 3\left(2 h_{2213}^{2}+h_{1123}^{2}+h_{2223}^{2}\right)+4\left(h_{1111}^{2}+h_{1112}^{2}\right) \\
& +h_{3332}^{2}+3\left(2 h_{1111} h_{3311}+h_{3311}^{2}+h_{3322}^{2}\right),
\end{aligned}
$$




$$
\begin{aligned}
\sum_{i, j, k} h_{i j k 3}^{2}= & \sum_{i} h_{i i i 3}^{2}+3 \sum_{i \neq k} h_{i i k 3}^{2} \\
= & h_{1113}^{2}+h_{2223}^{2}+h_{3333}^{2} \\
& +3\left(h_{1123}^{2}+h_{1133}^{2}+h_{2213}^{2}+h_{2233}^{3}+h_{3313}^{2}+h_{3323}^{2}\right) \\
= & 3\left(h_{1123}^{2}+h_{2213}^{2}\right)+h_{1113}^{2}+h_{2223}^{2} \\
& +3\left(\frac{a^{2}}{9 \lambda^{2}}+\frac{a^{2}}{9 \lambda^{2}}+\frac{4 a b}{9 \lambda^{2}}\right)+\frac{4 a^{2}}{9 \lambda^{2}} \\
= & 3\left(h_{1123}^{2}+h_{2213}^{2}\right)+h_{1113}^{2}+h_{2223}^{2}+\frac{10 a^{2}+12 a b}{9 \lambda^{2}}
\end{aligned}
$$

$$
\begin{aligned}
\text { LHS of }(*)= & \sum_{i, j, k} h_{i j k 1}^{2}+\sum_{i, j, k} h_{i j k 2}^{2}+\sum_{i, j, k} h_{i j k 3}^{2} \\
= & 12\left(h_{1123}^{2}+h_{2213}^{2}\right)+4\left(h_{1113}^{2}+h_{2223}^{2}\right) \\
& +8\left(h_{1111}^{2}+h_{1112}^{2}\right)+12 h_{1111} \frac{2}{3 \lambda}(a+b) \\
& +\frac{4 a b}{9 \lambda^{2}}+12\left[\frac{2(a+b)}{3 \lambda}\right]^{2}+\frac{10 a^{2}+12 a b}{9 \lambda^{2}} .
\end{aligned}
$$

Therefore,

$$
\frac{4}{\lambda^{2}} x+\frac{8}{\lambda^{2}} y+\frac{58 a^{2}+112 a b+48 b^{2}}{9 \lambda^{2}}=3 S(S-3)^{2}-10 S b,
$$

and the assertion follows since $S=6 \lambda^{2}$ and $S(S-3)=6 a+4 b$.

Unlike the Peng-Terng theorem, which can be established by studying $h$ and its covariant derivatives up to the second order at some distinguished point, we next have to appeal to $\nabla^{3} h$ since nothing can be drawn here, due to the lack of constraints among the quantities $x, y, a$, and $b$.

Now, on the one hand, by differentiating $\sum_{i, j} h_{i j}^{2}=S$ triply, we get

$$
h_{i j} h_{i j k l m}+h_{i j m} h_{i j k l}+h_{i j k} h_{i j l m}+h_{i j l} h_{i j k m}=0, \quad \forall k, l, m .
$$

Again, since at $p$,

$$
h_{i j} h_{i j k l m}=\lambda_{1} h_{11 k l m}+\lambda_{2} h_{22 k l m}+\lambda_{3} h_{33 k l m}=-3 \lambda h_{33 k l m},
$$

we have

$$
3 \lambda h_{33 k l m}=h_{i j m} h_{i j k l}+h_{i j k} h_{i j l m}+h_{i j l} h_{i j k m}, \quad \text { at } p, \forall k, l, m .
$$

It follows that at $p$,

$$
h_{k l m} h_{33 k l m}=\frac{1}{\lambda} h_{i j k} h_{k l m} h_{i j l m}
$$


On the other hand, by differentiating $\sum_{i, j, k} h_{i j k}^{2}=S(S-3)$ twice with respect to $e_{3}$, we have

$$
\sum_{i, j, k}\left(h_{i j k} h_{i j k 33}+h_{i j k 3}^{2}\right)=0, \quad \text { everywhere. }
$$

Recall that, at $p$,

$$
\sum_{i, j, k} h_{i j k 3}^{2}=\frac{x}{\lambda^{2}}+\frac{10 a^{2}+12 a b}{9 \lambda^{2}} .
$$

It follows that

(**) $h_{i j k}\left(h_{33 i j k}-h_{i j k 33}\right)=\frac{1}{\lambda} h_{i j k} h_{k l m} h_{i j l m}+\frac{x}{\lambda^{2}}+\frac{10 a^{2}+12 a b}{9 \lambda^{2}}$, at $p$.

Bearing in mind the results in Lemma $1, \nabla \delta_{i j}=0$ and

$$
R_{i j k l}=\left(1+\lambda_{i} \lambda_{j}\right)\left(\delta_{i k} \delta_{j l}-\delta_{i l} \delta_{j k}\right), \quad \text { at } p, \forall i, j, k, l,
$$

we compute

$$
\begin{aligned}
h_{i j k}\left(h_{33 i j k}-h_{i j k 33}\right)= & h_{i j k}\left[\nabla_{e_{k}}\left(h_{3 i j 3}+h_{m i} R_{m 33 j}+h_{3 m} R_{m i 3 j}\right)\right. \\
& \left.-\nabla_{e_{3}}\left(h_{i j 3 k}+2 h_{m j} R_{m i k 3}\right)\right] \\
= & h_{i j k}\left(h_{3 i j 3 k}-h_{3 i j k 3}+h_{m i k} R_{m 33 j}+3 h_{3 m k} R_{m i 3 j}\right) \\
& +h_{i j k} h_{m i} \nabla_{e_{k}}\left(h_{m 3} h_{3 j}-h_{m j} h_{33}\right) \\
& +h_{i j k} h_{3 m} \nabla_{e_{k}}\left(h_{m 3} h_{i j}-h_{m j} h_{i 3}\right) \\
& -2 h_{i j k} h_{m j} \nabla_{e_{3}}\left(h_{m k} h_{i 3}-h_{m 3} h_{i k}\right) \\
= & h_{i j k}\left(2 h_{m i j} R_{m 33 k}+5 h_{3 m j} R_{m i 3 k}\right) \\
& +h_{i j k} h_{m i}\left(h_{m 3 k} h_{3 j}+h_{m 3} h_{3 j k}-h_{m j k} h_{33}\right) \\
& +h_{i j k} h_{3 m}\left(h_{m 3} h_{i j k}-h_{m j k} h_{i 3}-h_{m j} h_{i 3 k}\right) \\
& -2 h_{i j k} h_{m j}\left(h_{m k 3} h_{i 3}-h_{m 3} h_{i k 3}\right) \\
= & \sum_{i, j, k, m}\left[2 h_{i j k} h_{m i j}\left(1+\lambda_{3} \lambda_{k}\right)\left(\delta_{m 3} \delta_{3 k}-\delta_{m k}\right)\right. \\
& \left.+5 h_{i j k} h_{3 m j}\left(1+\lambda_{3} \lambda_{k}\right)\left(\delta_{m 3} \delta_{i k}-\delta_{m k} \delta_{i 3}\right)\right] \\
& +\sum_{i, k} \lambda_{3} \lambda_{i} h_{i k 3}^{2}+\sum_{i, k} \lambda_{3}^{2} h_{i k 3}^{2}-\sum_{i, j, k} \lambda_{3} \lambda_{i} h_{i j k}^{2} \\
& +\sum_{i, j, k} \lambda_{3}^{2} h_{i j k}^{2}-\sum_{j, k} \lambda_{3}^{2} h_{j k 3}^{2}-\sum_{i, j} \lambda_{3}^{2} h_{i k 3}^{2}
\end{aligned}
$$




$$
\begin{aligned}
& -\sum_{j, k} 2 \lambda_{3} \lambda_{j} h_{j k 3}^{2}+\sum_{j, k} 2 \lambda_{3}^{2} h_{j k 3}^{2} \\
& =\sum_{i, j} 2\left(1+\lambda_{3}^{2}\right) h_{i j 3}^{2}-\sum_{i, j, k} 2\left(1+\lambda_{3} \lambda_{k}\right) h_{i j k}^{2} \\
& -\sum_{j, k} 5\left(1+\lambda_{3} \lambda_{k}\right) h_{j k 3}^{2}-\sum_{i, k} \lambda_{3} \lambda_{i} h_{i k 3}^{2} \\
& +\sum_{i, k} \lambda_{3}^{2} h_{i k 3}^{2}-\sum_{i, j, k} \lambda_{3} \lambda_{i} h_{i j k}^{2}+\sum_{i, j, k} \lambda_{3}^{2} h_{i j k}^{2} \\
& =2\left(1+4 \lambda^{2}\right)(2 a)-2(6 a+4 b)+4 \lambda(4 \lambda b) \\
& -5(2 a)+10 \lambda(2 \lambda a)+2 \lambda(2 \lambda a)+4 \lambda^{2}(2 a) \\
& +2 \lambda(4 \lambda b)+4 \lambda^{2}(6 a+4 b) \\
& =\left(72 \lambda^{2}-18\right) a+\left(40 \lambda^{2}-8\right)^{b} \text {, } \\
& h_{i j k} h_{k l m} h_{i j l m}=h_{k l m}\left(h_{11 k} h_{11 l m}+h_{22 k} h_{22 l m}+2 h_{12 k} h_{12 l m}\right. \\
& \left.+2 h_{13 k} h_{13 l m}+2 h_{23 k} h_{23 l m}\right) \\
& =h_{11 k} h_{k l m}\left(h_{11 l m}-h_{22 l m}\right) \\
& +2\left(h_{112} h_{1 l m}-h_{111} h_{2 l m}\right) h_{12 l m} \\
& +2 h_{113} h_{1 l m} h_{13 l m}-2 h_{113} h_{2 l m} h_{23 l m} \\
& =h_{11 k}\left[h_{k 11}\left(h_{1111}-h_{2211}\right)+h_{k 22}\left(h_{1122}-h_{2222}\right)\right. \\
& +2 h_{k 12}\left(h_{1112}-h_{2212}\right)+2 h_{k 13}\left(h_{1113}-h_{2213}\right) \\
& \left.+2 h_{k 23}\left(h_{1123}-h_{2223}\right)\right] \\
& +2 h_{112}\left(h_{111} h_{1211}+h_{122} h_{1222}+2 h_{112} h_{1212}+2 h_{113} h_{1213}\right) \\
& -2 h_{111}\left(h_{211} h_{1211}+h_{222} h_{1222}+2 h_{122} h_{1212}+2 h_{223} h_{1223}\right) \\
& +2 h_{113}\left[h_{111} h_{1113}+h_{122} h_{1322}+2 h_{112} h_{1312}\right. \\
& \left.+h_{113}\left(h_{1133}+h_{3311}\right)\right] \\
& -2 h_{113}\left[h_{211} h_{1123}+h_{222} h_{2322}+2 h_{212} h_{2312}\right. \\
& \left.+h_{223}\left(h_{2233}+h_{3322}\right)\right] \\
& =2(a+b)\left(h_{1111}-h_{2211}\right)+4 b h_{1122} \\
& -4 h_{113}\left(h_{111} h_{3313}+h_{112} h_{3323}\right) \\
& +2 a\left(h_{1133}+h_{3311}+h_{2233}+h_{3322}\right) \\
& =4 a h_{1111}+2(a-b) \frac{2(a+b)}{3 \lambda}-4 a \frac{2 b}{3 \lambda}+4 a \frac{a+2 b}{3 \lambda} \\
& =4 a h_{1111}+\frac{8 a^{2}-4 b^{2}}{3 \lambda} \text {. }
\end{aligned}
$$


Substituting all the above results to $(* *)$, we have

$$
\left(72 \lambda^{2}-18\right) a+\left(40 \lambda^{2}-8\right) b=\frac{4 a h_{1111}}{\lambda}+\frac{8 a^{2}-4 b^{2}}{3 \lambda^{2}}+\frac{x}{\lambda^{2}}+\frac{10 a^{2}+12 a b}{9 \lambda^{2}} .
$$

That is,

$$
x+4 a \lambda h_{111}=-\frac{34}{9} a^{2}-\frac{4}{3} a b+\frac{4}{3} b^{2}+\lambda^{2}\left(72 \lambda^{2}-18\right) a+\lambda^{2}\left(40 \lambda^{2}-8\right) b .
$$

We are now in a position to conclude the Main Theorem. Recall that from Lemma 2, at $p$

$$
x+2 \lambda^{2}\left(h_{1111}^{2}+h_{1112}^{2}\right)+2(a+b) \lambda h_{1111}=\frac{26}{9} a^{2}+\frac{7}{18} a b-b^{2}-\frac{5}{4} S b .
$$

By eliminating $x$ from the above two equations, we have $(t)$

$$
\begin{aligned}
& 2 \lambda^{2}\left(h_{1111}^{2}+h_{1112}^{2}\right)-2(a-b) \lambda h_{1111} \\
& \quad=\frac{20}{3} a^{2}+\frac{31}{18} a b-\frac{7}{3} b^{2}-\frac{5}{4} S b-\lambda^{2}\left(72 \lambda^{2}-18\right) a-\lambda^{2}\left(40 \lambda^{2}-8\right) b .
\end{aligned}
$$

Since $\lambda^{2}=\frac{S}{6}$ and $S(S-3)=6 a+4 b$,

$$
\begin{aligned}
\text { RHS of }(\dagger) & =\frac{20}{3} a^{2}+\frac{31}{18} a b-\frac{7}{3} b^{2}-\frac{5}{4} S b-\frac{S}{6}(12 S-18) a-\frac{S}{6}\left(\frac{20}{3} S-8\right) b \\
& \leq \frac{20}{3} a^{2}+\frac{31}{18} a b-\frac{7}{3} b^{2}-2 S(S-3) a-\frac{10}{9} S(S-3) b \\
& =\frac{20}{3} a^{2}+\frac{31}{18} a b-\frac{7}{3} b^{2}-2(6 a+4 b) a-\frac{10}{9}(6 a+4 b) b \\
& \leq-\frac{7}{3}\left(a^{2}+b^{2}\right) .
\end{aligned}
$$

It is clear that

$$
\text { LHS of }(\dagger) \geq-\frac{1}{2}(a-b)^{2} \geq-\frac{1}{2}\left(a^{2}+b^{2}\right),
$$

which yields $a=b=0$ as desired and completes the proof of the Main Theorem.

\section{Acknowledgment}

The author wishes to thank Y. Xu for his interest in this work, and to express indebtedness to $\mathrm{S}$. Y. Cheng for his valuable suggestions and continuous advice, support and encouragement. 


\section{References}

[1] E. Cartan, Sur des familles remarquables d'hypersurfaces isoparametriques dans les espaces spheriques, Math. Z. 45 (1939) 335-367.

[2] T. E. Cecil \& P. J. Ryan, Tight and taut immersions of manifolds, Pitman, 1985.

[3] S. Chang, On isoparametric hypersurfaces of rank 4 in spheres, in preparation.

[4] S. S. Chern, M. do Carmo \& S. Kobayashi, Minimal submanifolds of a sphere with second fundamental form of constant length, Functional Analysis and Related Fields, Springer, Berlin, 1970, 59-75.

[5] S. De Almeida \& F. Brito, Closed 3-dimensional hypersurfaces with constant mean curvature and constant scalar curvature, Duke Math. J. 61 (1990) 195-206.

[6] H. B. Lawson, Local rigidity theorems for minimal hypersurfaces, Ann. of Math. (2) 89 (1969) 187-191.

[7] C. K. Peng \& C. L. Terng, Minimal hypersurface of spheres with constant scalar curvature, Annals of Math. Studies, No. 103, Princeton University Press, Princeton, NJ, 1983, 177-198.

[8] __, The scalar curvature of minimal hypersurfaces in spheres, Math. Ann. 266 (1983) 105-113.

[9] J. Simons, Minimal varieties in a Riemannian manifold, Ann. of Math. (2) 88 (1968) 62-105.

[10] S. T. Yau, Submanifolds with constant mean curvature. II, Amer. J. Math. 97 (1975) 76-100.

[11] _ _ Problem section, Annals of Math. Studies, No. 102, Princeton University Press, Princeton, NJ, 1982, 693.

UNiversity of CALIFornia, Los ANGeles 